\title{
Estimating the incidence of occupational asthma and rhinitis from laboratory animal allergens in the UK, 1999-2000
}

\section{A Draper, A Newman Taylor, P Cullinan}

We report a survey of the number of persons handling laboratory animals in the UK in 1999-2000. This estimate has allowed us to calculate annual incidence rates of allergic asthma and rhinitis attributable to laboratory animals, insects, and birds using numerators derived from cases reported to a national surveillance scheme (SWORD). The number of exposed employees has almost halved since the early 1980s. From these data, the estimated annual incidence rate of occupational asthma among persons working with small mammals is $1.56 / 1000$ employees. Although reported cases are rare, estimated incidence rates of asthma attributable to birds (23.9/1000/year) and insects (2.35/1000/year) are high.

aboratory animal allergens are an important cause of occupational asthma in the UK. A national surveillance - scheme (SWORD, The Surveillance of Work \& Occupational Related Diseases) has recorded new cases of occupational asthma and rhinitis in the United Kingdom, annually since 1989. The scheme relies on voluntary reporting of incident cases, with details of agent and job, by respiratory and occupational physicians. A core set of physicians reports on a monthly basis; others are randomly sampled for one month of each year. The numbers of new cases of occupational lung disease are estimated each year, taking into account the sampling fractions; and occupation specific rates are calculated using denominators derived from the Labour Force Survey. The population of laboratory assistants and technicians

\section{Main messages}

- The number of persons in contact with small laboratory animals in the UK has halved since the last estimate in 1981.

- The data suggest that the incidence of occupational asthma attributable to small animal species may be higher than recent estimates.

- The rates of asthma attributable to birds and insects during 1999/2000, although based on small numbers, were high.

\section{Policy implications}

- Occupational health physicians involved in the care of those working with small animal species should remain especially vigilant against the development of occupational asthma. was estimated as 127478 in $1989,{ }^{1}$ and the estimated annual incidence of occupational asthma in this category was 0.235/1000/year from 1989 to $1991 .^{2}$ However, only a proportion of this population works with laboratory animals. Over the past 20 years there has been a steady reduction in the number of animal experiments in the UK, ${ }^{3}$ and in consequence the number of employees currently in contact with laboratory animals is likely to be much lower than the previous estimate of 32000 in $1981 .{ }^{4}$ We aimed to determine the number of individuals having current contact with laboratory animals and to use this as the denominator to estimate the incidence of occupational asthma among this group of employees.

\section{METHODS}

Every institution in the United Kingdom that undertakes animal experimentation is required to nominate an individual (the holder of certificate of designation) who oversees all aspects of the procedures. ${ }^{5}$ They, numbering 296 in 1999, are each registered with the Home Office. We devised a single page questionnaire in which respondents were asked to enumerate the employees at their institution by job category and species handled. Three broad job categories were considered: animal technicians, researchers/scientists, and others (maintenance, security, etc). Species were grouped into small (rats, mice, guinea pigs, hamsters, ferrets, and rabbits) and large (cats, dogs, sheep, cows, primates, horses, goats, and pigs) animals. Groups for insects and other animals were also included. The Home Office delivered the questionnaire with a circular during October 1999 and anonymous responses were returned to the authors in a reply envelope. Two follow up mailings were conducted in early 2000. The estimated range of the total population, our denominator, is based on the information obtained and the likely characteristics of the non-responding populations. The lower estimates reflect the actual responses for small animals and assume that none of the non-responders used them at their sites. The higher estimates assume that the distribution among non-responders is the same as the responders. Quarterly SWORD reports over 12 months following the survey identified the number of new cases of occupational asthma and rhinitis reported among laboratory staff which were attributed to laboratory animals, insects, or birds. We calculated numerators (with 95\% confidence intervals based on a Poisson distribution) using the sampling fraction for four consecutive quarters. Incidence rates were calculated using the higher estimates of the denominator population. The data were analysed using STATA (Stata Corporation, Texas).

\section{RESULTS}

Most of the surveyed sites were commercial organisations $(38 \%)$ or places of higher education (34\%). A total of 207 


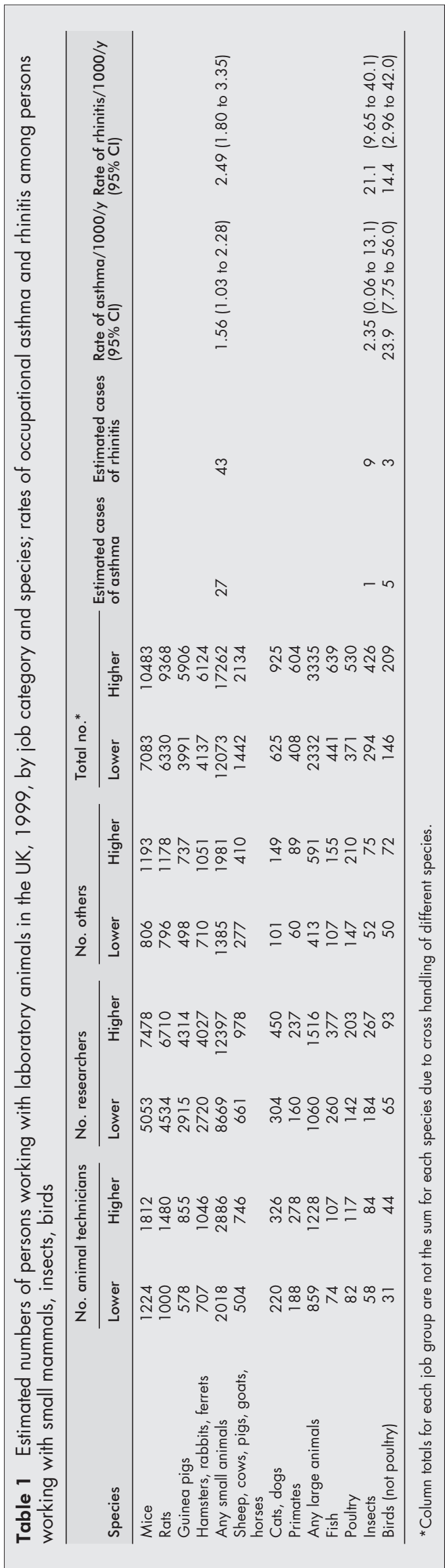

questionnaires (70\% of those eligible) were returned; information regarding the breakdown of species for small and large animals was available for 200 responses. Table 1 shows the lower and higher estimated numbers of employees grouped by species and job category. Between 12000 and 17300 employees were working with small mammals, in most cases scientists working with mice or rats. Much smaller numbers work with larger animals, birds, insects, or fish.

Using the higher estimates of the denominators, and among those working with small animals, we calculated a current, annual incidence rate of 1.56 cases/1000 persons for occupational asthma and a figure of $2.54 / 1000$ persons for occupational rhinitis. The rates for those working with birds or fish were higher, but based on a small number of cases.

\section{DISCUSSION}

This is the first attempt in 20 years to determine the size of the population occupationally exposed to laboratory animals. Over this period major changes have taken place in the number and type of animal experiments conducted in the UK. Our results suggest that the number of exposed employees has halved since 1981. Unlike the earlier estimate, we have been able also to estimate the numbers working with individual species. The higher of our estimates of those working with small animals is in close agreement with the number of current personal licence holders (animal technicians and researchers, $\mathrm{n}=13700$ ) recorded by the Home Office in 1999. In 2000 there were 1900 animal technicians who were members of the Institute of Animal Technology, ${ }^{6}$ a figure also close to our lower estimate. In future, these more readily available statistics may serve as a useful indicator for these populations. Our estimated rates of occupational asthma and rhinitis are high; but nonetheless are likely to underestimate the true figures because the numerator depends on cases reported by chest and occupational physicians to SWORD. Rates among newer employees, who are probably at particularly high risk, may also be higher.

\section{ACKNOWLEDGEMENT}

We are grateful to the Home Office for their help with this survey; and to each of those who responded to our questionnaire.

\section{Authors' affiliations}

A Draper, A Newman Taylor, P Cullinan, Department of Occupational \& Environmental Medicine, Royal Brompton Hospital \& $\mathrm{NHLI}$, London, UK

Correspondence to: Dr P Cullinan, Department of Occupational \& Environmental Medicine, Royal Brompton Hospital \& NHLI, lb Manresa Road, London SW3 6LR, UK; p.cullinan@ic.ac.uk

Accepted 11 October 2002

\section{REFERENCES}

1 McDonald JC, Keynes HL, Meredith SK. Reported incidence of occupational asthma in the United Kingdom, 1989-97. Occup Environ Med 2000:57:823-9.

2 Meredith SK, Taylor VM, McDonald JC. Occupational respiratory disease in the United Kingdom 1989: a report to the British Thoracic Society and the Society of Occupational Medicine by the SWORD project group. Br J Ind Med 1991;48:292-8.

3 Cockcroft A, Edwards J, McCarthy P, et al. Allergy in laboratory animal workers. Lancet 1981;1:827-30.

4 Home Office. Press release, July 1999, http://www.apc.gov.uk/ press_releases/990723.htm.

5 Home Office. Draft Guidance on the operation of the Animals (Scientific Procedures) Act 1986, http://www.homeoffice.gov.uk/animact/ aspileaf.htm.

6 Institute of Animal Technology, Minutes of annual general meeting, 2001, http://www.iat.org.uk/. 